\title{
Primary spontaneous cerebrospinal fluid rhinorrhoea ${ }^{1}$
}

\author{
JOHN E. A. O'CONNELL
}

From St. Bartholomew's Hospital, London

The adjective 'spontaneous' as applied to cerebrospinal fluid rhinorrhoea simply indicates that the discharge is not an immediate or delayed result of trauma. Cases of spontaneous cerebrospinal fluid rhinorrhoea can be subdivided into two distinct groups, secondary and primary. In the secondary group an obvious cause for the leak is present. This may be some gross lesion producing erosion of the skull base; examples of such lesions are osteomata of the nasal sinuses, intracranial neoplasms, particularly pituitary adenoma and craniopharyngioma, and internal hydrocephalus due to neoplastic or other cause; the lesion may be a meningocele or or meningo-encephalocele, the rhinorrhoea at times following attempts to treat the swelling by an intranasal operation. In the primary group no cause for the leak of cerebrospinal fluid can be found, and it is with this group of unknown aetiology that the present communication is concerned.

As is the case in all medical problems, so in that of cerebrospinal fluid rhinorrhoea adequate knowledge of the relevant anatomy and physiology is essential if abnormalities are to be recognized and understood. In the second century Galen had observed the fluid in the ventricles of the brain and considered it a waste product to be excreted via the pituitary into the nose. Vesalius and the other great anatomists of the middle ages also mentioned the fluid and in the seventeenth and eighteenth centuries Willis and Cotugno studied it. Probably it was the publication of the results of his researches, carried out over a period of 15 years, by Magendie in 1842 that contributed most to a general understanding of the anatomy and physiology of the cerebrospinal fluid. The post-traumatic discharge of fluid from the nose had been described by Bidloo the elder (quoted by St Clair Thomson, 1899) in the seventeenth century. The first description of what was certainly spontaneous cerebrospinal fluid rhinorrhoea was given by Miller in 1826 . He gives the history of a boy with a progressively enlarging head who developed a recurring discharge of fluid from his nose and in whom a large internal hydrocephalus was revealed at necropsy;

\footnotetext{
'A paper read at the combined meeting of the Neurosurgical Society of America and the Society of British Neurological Surgeons in London in June 1963.
}

the route by means of which the fluid reached the nose from the cranium was also recognized, an opening to the right of and anterior to the crista galli. In $1899 \mathrm{St}$ Clair Thomson reviewed the literature dealing with spontaneous cerebrospinal fluid rhinorrhoea up to that date, describing 21 cases, including one of his own. The reports reviewed had appeared between 1834 and 1898. Study of these case records suggests that in possibly nine the rhinorrhoea was of unknown aetiology; in the others evidence of a lesion to which the leak was secondary appears undoubted. Johnston in 1926 reviewed 21 cases reported before that date and after the publication of St Clair Thomson's monograph. In eight of these the rhinorrhoea appeared to be a primary one. Coleman and Troland (1947) collected another 12 examples of the primary type and added three personal cases. Before 1954 other reports of one or two cases of cerebrospinal fluid rhinorrhoea with no apparent cause had been presented by Wessels (1939, one case), Adson (1941, one case), Love and Gay (1947, two cases), Cloward and Cunningham (1947, one case), and Rand (1953, two cases). This makes a total of only 39 cases reported before 1954 . No doubt this list of published cases is incomplete and others have been added in the last 10 years. It seems likely, however, that the number of cases reported to date is less than 75 .

All those who have studied the subject of primary cerebrospinal fluid rhinorrhoea have given thought to two particular problems: first the force responsible for the escape of the fluid, and secondly the route by which it enters the nose. Concerning the force responsible, Coleman and Troland considered it unknown, but other authors (St Clair Thomson, Johnston, Locke, Love and Gay, Rand) have regarded raised intracranial pressure, long-continued or transient but recurring, to be an essential feature. In discussing the route of entry of cerebrospinal fluid into the nose, St Clair Thomson suggested as possibilities a small intranasal meningocele, a communication between subarachnoid space and nasal lymphatics, or an imperceptible opening in the cribriform plate. Johnston suggested a persistent craniopharyngeal duct as a possible channel but Locke (1926) considered that the opening was invariably in the cribri- 
form plate. Coleman and Troland thought that the fluid spread along the arachnoid sheaths of the olfactory nerves, while Rand believed that a dysplasia of either the developing cartilaginous cribriform plate or of the meninges related to the olfactory nerves caused a fistula through which a leak became established.

Two personal cases of primary spontaneous cerebrospinal fluid rhinorrhoea will now be described.

\section{CASE REPORTS}

CASE 1 Mrs. O.B., aged 42, a housewife and mother of four children, was referred by Dr. K. Latter and admitted on 3 December 1954, complaining of a discharge from the right nostril. This had begun 15 months previously. There had been no preceding head injury, nor was the onset of the discharge related to any form of heavy physical exertion. At first the clear fluid escaped as a drop every five minutes, but the flow increased gradually until on bending forwards the dripping was continuous with intervals of a few seconds between drops. The patient became aware of a salty taste in her mouth and was occasionally awakened at night by the accumulation of the fluid in her throat. Her only other symptoms were a mild retro-orbital headache and a deterioration of her sense of taste and smell. There was nothing of significance in her past or family history.

Examination revealed a healthy looking woman with but two abnormalities on general physical and neurological examination; first a colourless watery discharge from the

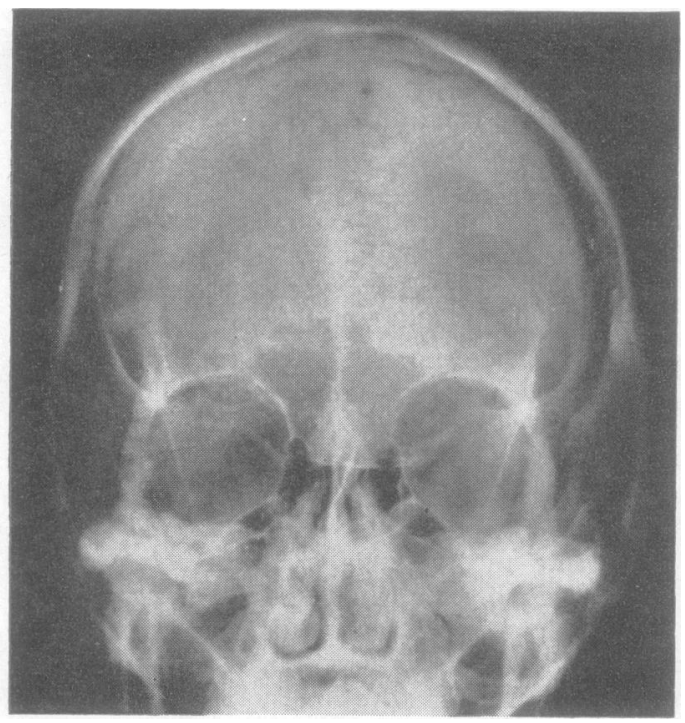

FIG. 1a. right nostril when the head was flexed (the drops were separated by an interval of some 10 seconds and escaped 으 more rapidly during jugular compression); secondly $Z$ there was a severe bilateral reduction in the sense of smell. Examination of the nasal discharge showed a protein content of $20 \mathrm{mg}$., chlorides $760 \mathrm{mg}$., and glucose $75 \mathrm{mg}$. per $100 \mathrm{ml}$. with no cells. Other laboratory investigations 0 revealed no abnormality. The Wassermann reaction was ${ }_{\mathcal{D}}^{0}$ negative. Skull films were normal; there was no fracture nor any abnormality of the cribriform area (Figure 1). The diagnosis was thus one of spontaneous cerebrospinal fluid rhinorrhoea of unknown aetiology.

At operation a coronal incision was made and the scalp $\Rightarrow$ flap reflected anteriorly, a bifrontal block of bone being $?$ elevated (Figure 2). The dura mater was incised at the anterior limit of the exposure and the sagittal sinus divided $\bar{\sigma}$ between ligatures. The right frontal lobe was elevated $\frac{\bar{T}}{\frac{9}{5}}$ from the floor of the anterior fossa and no suggestion $\mathbb{\Phi}$ of a dural tear or old fracture was seen. A loose arachnoid fold was present along the medial and inferior margin of $\omega$ the lobe and passed onto the superior aspect of the right $\overrightarrow{0}$ olfactory bulb. No such arrangement of the arachnoid was present on the left side. When this fold of arachnoid $\vec{\omega}$ was divided it was seen (Fig. 2) that the olfactory bulb had atrophied in its anterior half beneath the area of attachment of the fold, and exposed on the cribriform 0 plate was a punched-out circular dural opening through ${ }^{\prime}$ which a $1 \mathrm{~mm}$. probe could be passed into the nasa $\omega$ cavity. The olfactory bulb and anterior tract were excised in and a patch of temporal fascia sutured to the basal duf \pm to cover the whole of the cribriform plate and its immedi-응 ate environment. After suture of the dural incision the bone block was replaced and the wound closed. Post- $\vec{c}$
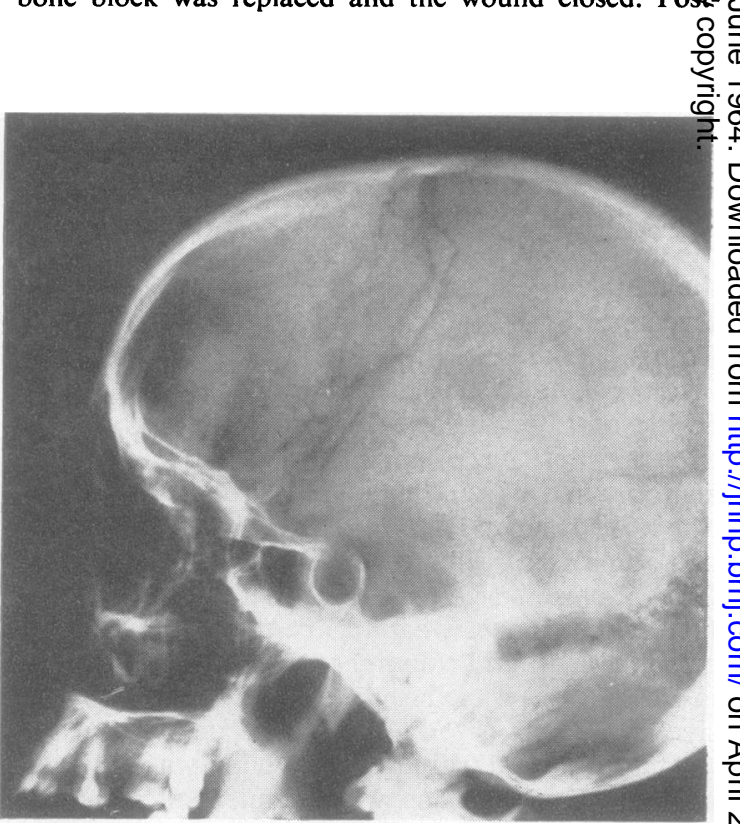

FIG. $1 \mathrm{~b}$. 


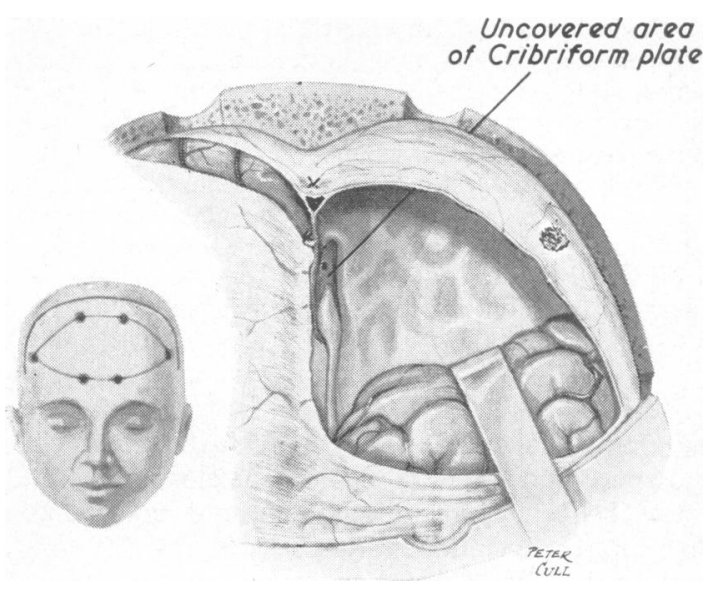

FIG. 2. Sketch to demonstrate the appearances at operation in case 1 .

operatively the rhinorrhoea stopped immediately and there has been no recurrence. The patient has a right anosmia but the sense of smell has perhaps improved on the left. Five years later the patient was delivered of a baby son and remains in perfect health at the present time (May, 1963).

CASE 2 Mr. K.A., aged 44, a Negro machinist, was admitted on 28 May 1962, being referred by Dr. Aldren Turner because of a copious watery discharge from his left nostril. He stated that some six months previously he had had a clear discharge from his nose for a couple of days. There was no further discharge until seven weeks before admission when it recurred and persisted. Its onset was not associated with injury to the head or physical exertion. The discharge was copious and when he lay down he was conscious of the fluid running into the pharynx. He stated that eight years previously he had been involved in a lorry accident; although the driver was killed he had not himself sustained any head or other injury. For a few years he had suffered from grand mal epilepsy and shown a personality change. There were no other symptoms and the family history contributed nothing.

On examination he was alert and cooperative. On tilting his head forward there was a rapid discharge of clear fluid from the left nostril, its rate being increased by jugular compression. Neurological examination showed no abnormality apart from a possible reduction of the sense of smell. Examination of the nasal discharge revealed that it was cerebrospinal fluid. On general examination the only abnormality was an irregularly recurring pyrexia associated with a 16,000 leucocytosis (93\% polymorphs). Both the fever and the leucocytosis subsided with penicillin therapy. The cerebrospinal fluid obtained at lumbar puncture showed no abnormality, the protein being normal. The Wassermann reaction was negative in the blood and cerebrospinal fluid and other laboratory investigations were also negative. Skull films

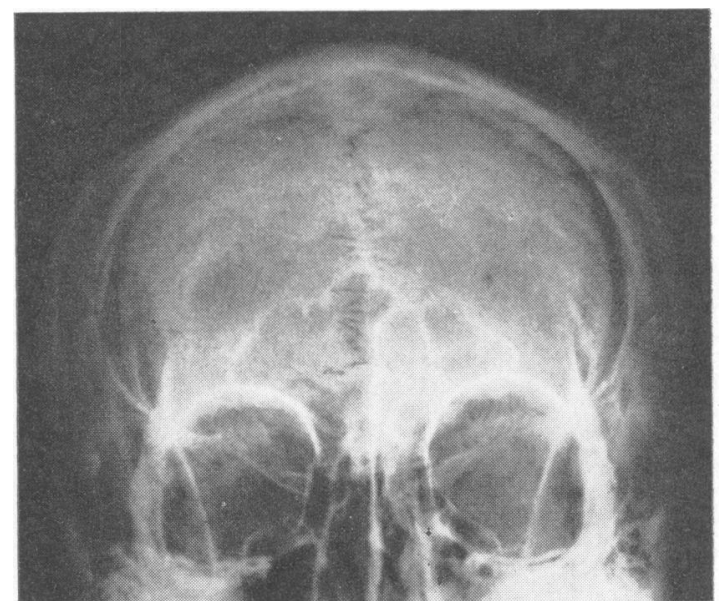

FIG. 3a.

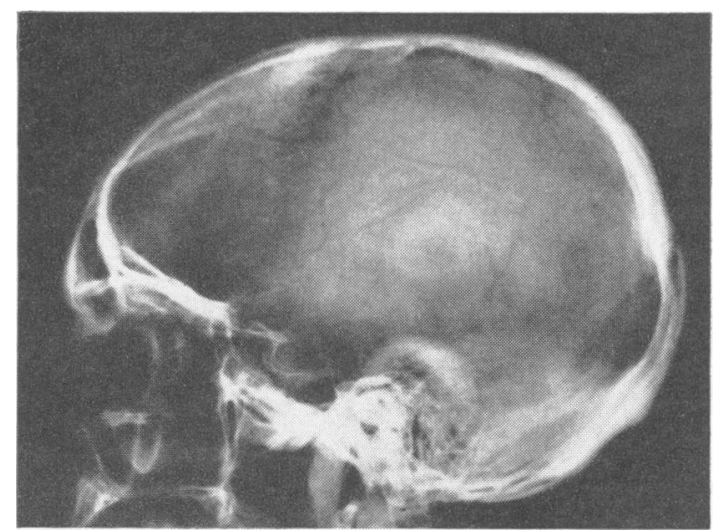

FIG. $3 b$.

FIs. 3a and b. Skull films of case 2 .

showed no abnormality other than an opacity of the left ethmoid and maxillary sinuses, possibly due to cerebrospinal fluid within them (Figure 3). Pneumoencephalography showed no evidence of an internal hydrocephalus or tumour in the sellar region or elsewhere. The diagnosis was considered to be one of spontaneous cerebrospinal fluid rhinorrhoea of unknown aetiology and exploration was decided upon. A left frontal osteoplastic flap was made and the frontal lobe elevated without revealing any adhesions between it and the orbital roof or anterior sphenoidal region, the sella and both optic nerves being visualized. It was noted that a loose funnel of arachnoid passed forward around each olfactory tract (Figure 4). The left tract was divided and excised with the olfactory bulb, revealing a round opening in the cribriform plate through which a fine probe could be passed into the nose. The further abnormality now noted was that the cribriform plate was situated at the bottom of a deep cleft between the falx and crista galli medially and the strongly arched orbital roof laterally. A piece of temporal muscle 


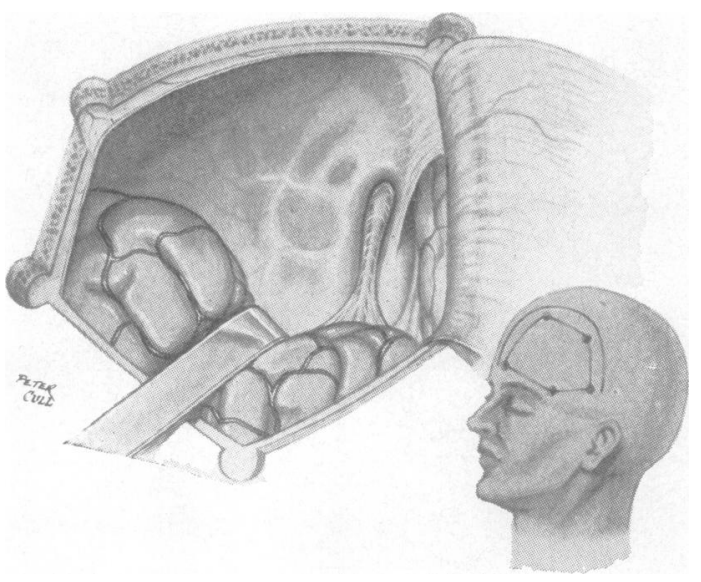

FIG. 4. Sketch to demonstrate appearances at operation in case 2. was plugged into this cleft onto the cribriform plate. Thes same congenital abnormality on the right side was dealt $\bar{Z}$ with similarly and then the whole area on both sides of theD midline covered with a sheet of temporal fascia which was sutured in position. The wound was then closed. Postoperatively a small leak of cerebrospinal fluid was notecic on several occasions over a few days. This then ceased and there has been no recurrence. One year post-更 operative by the patient continues to have occasional epileptic attacks but is otherwise entirely well.

\section{DISCUSSION}

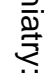

$\overrightarrow{\overrightarrow{\vec{C}}}$

The anatomical relationship between the subarach noid space and the nasal cavity is a close one. Theo thin cribriform plate of the ethmoid is covereds superiorly by dura mater and inferiorly by olfactory mucosa. In the photomicrograph reproduced im Fig. 5 it will be seen that the measurement from the internal surface of the dura mater to the nasal surface

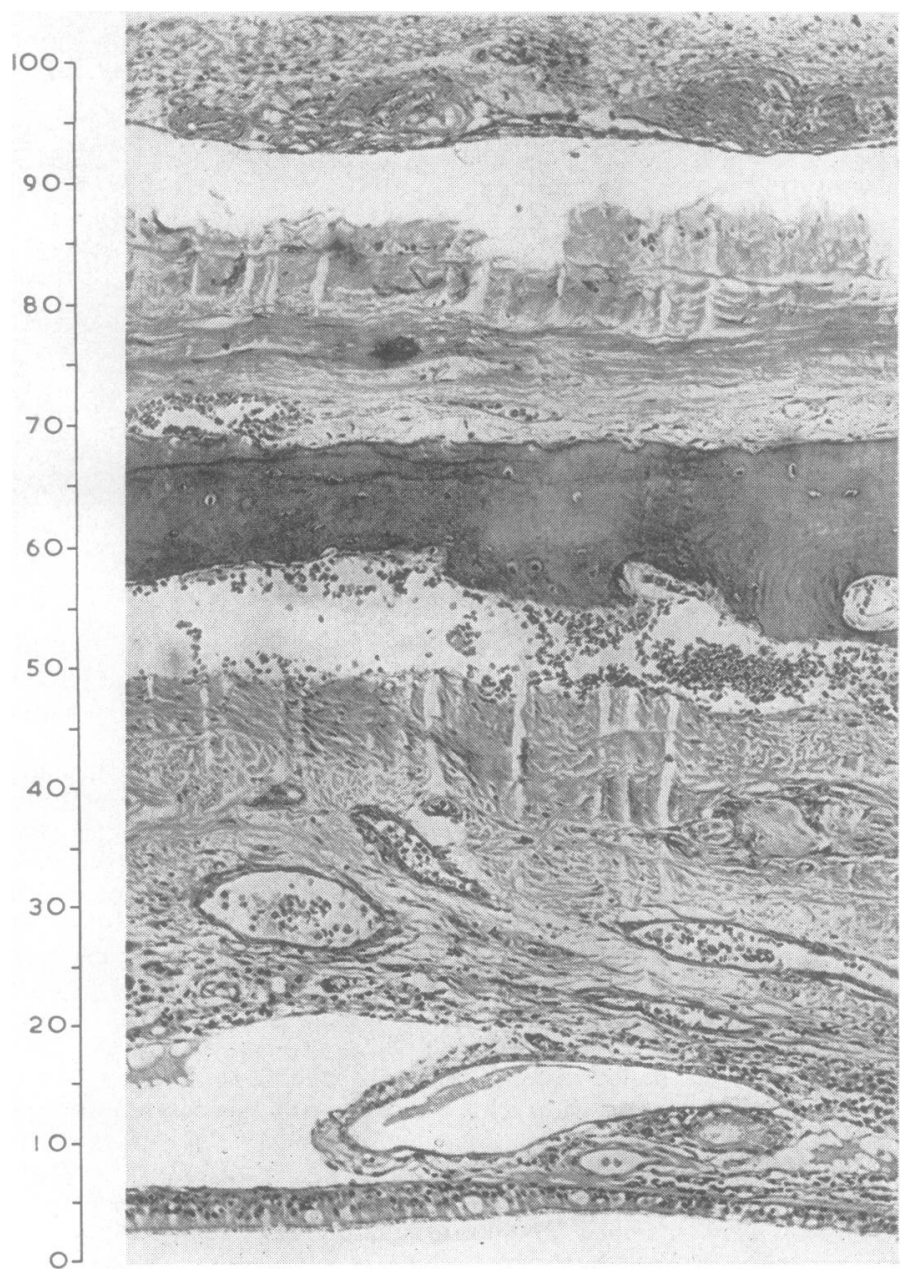

FIG. 5. Photomicrograph of sec $\bumpeq$ tion through lamina cribrosa. Each small division on the scale measures one hundredth of a millimetre. 
of the mucosa is $0.85 \mathrm{~mm}$.; correction for shrinkage occasioned by fixation would suggest that the measurement would be $1.1 \mathrm{~mm}$. The cribriform plate is pierced by 10 to 20 olfactory nerves en route from the olfactory mucosa to bulb. Each of these nerves is surrounded by a prolongation of dura mater which fuses inferiorly with its epineurium; within the dural sheath is an arachnoid prolongation fusing inferiorly with the pial sheath of the nerve. There is thus a prolongation of the subarachnoid space into, and at times through, the foramina in the lamina cribrosa and it seems probable that the tissue barrier between subarachnoid space and nasal cavity is frequently but a fraction of a millimetre in thickness.

The possibility that, as a normal physiological process, cerebrospinal fluid drained through the cribriform plate into the nose was accepted by Willis though denied by Vieussens. Later the animal experiments of Schwalbe (1869), of Key and Retzius (1875), and of Weed (1914) revealed that fine particles injected into the subarachnoid space passed into the nasal submucosa and thence to lymphatic vessels. It is generally accepted that cerebrospinal fluid is absorbed by this route. Le Gros Clark (1929) demonstrated that dye injected into the nasal cavity of rabbits entered the cranium along the olfactory nerves and indeed it has been thought that this may be the route by which certain infections reach the nervous system.

It is clear that the anatomical relationship between the subarachnoid space and the surface of the body is more intimate at the lamina cribrosa than at any other situation. It might perhaps be expected that, in the absence of gross disease, the thin barrier between the cerebrospinal-fluid-filled spaces on the one hand and the nasal cavity on the other could at times prove inadequate, and a communication between the two become established, cerebrospinal fluid rhinorrhoea resulting. Normally the superior surface of the cribriform plate is entirely covered by the olfactory bulb. The inferior surface of the frontal lobe is closely applied to the floor of the anterior fossa and to the olfactory bulb and the weak spot on the cranial floor is thus well covered. However, the covering may at times be defective. Thus in case 1 the anterior half of the olfactory bulb had atrophied and the corresponding area of the cribriform plate lay at the bottom of a funnel of frontal lobe arachnoid adherent around its margins (Figure 2). In this exposed area was a rounded opening through which a probe could be passed into the nose. It is believed that for some reason atrophy of a portion of the olfactory bulb occurred here as the primary event, and that the pressure of the cerebrospinal fluid on the exposed lamina cribrosa was the factor leading to distention of the meningeal pouch in an empty

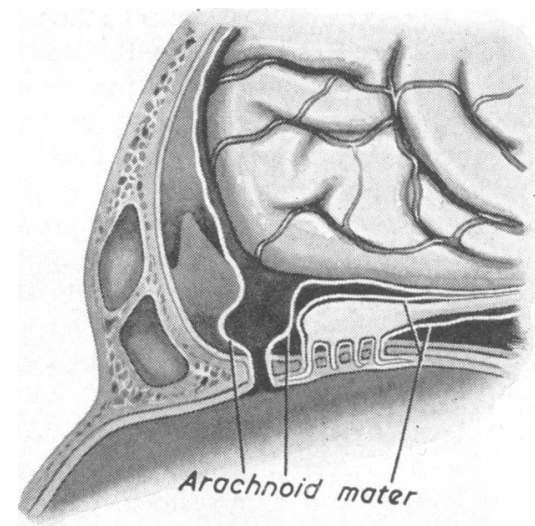

FIG. 6. Sketch to demonstrate anatomy of the fistula in case 1 .

neural foramen and the eventual establishment of a fistula (Figure 6). In case 2 two abnormalities were noted. First a loose funnel of arachnoid passed forwards around each olfactory tract and onto the bulb, an arrangement quite different from the usual, in which the arachnoid closely invests the tract and bulb (Figure 4). Secondly there was a deformity of the skull base which resembled that noted at operation in at least four of 10 cases of spontaneous cerebrospinal fluid rhinorrhoea recorded in the literature since 1939. This deformity consisted in the presence of a deep narrow cleft between the strongly arched orbital roof laterally and the crista galli and

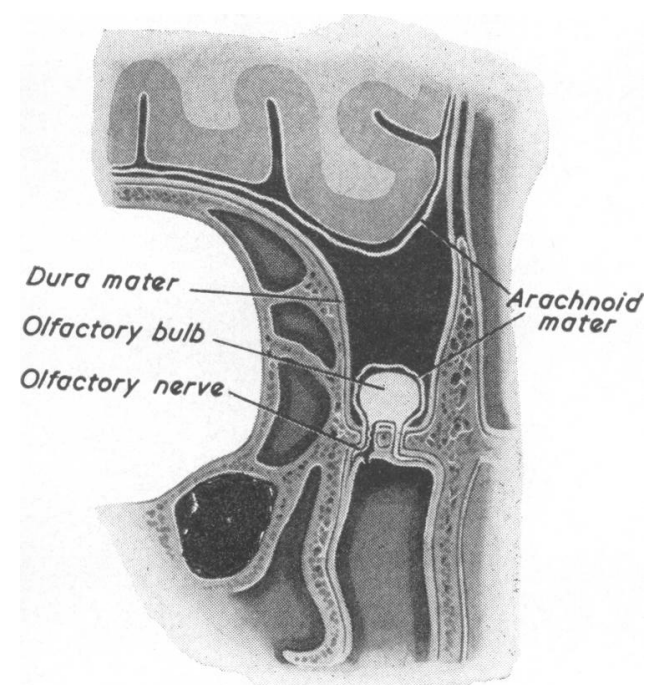

FIG. 7. Sketch to demonstrate the anatomy of the fistula in case 2. 
falx medially, with the olfactory bulb sunken on its floor (Figure 7). It is suggested that the deformity removed the olfactory bulb and tract from pressure from the overlying frontal lobe and that their arachnoid sheath therefore remained widely open. As a result, a wide subarachnoid cistern lies upon the superior surface of the lamina cribrosa and prolongation of this, the arachnoid sheaths of the olfactory nerves, are distended by the normal cerebrospinal fluid pressure. Secondary enlargement and intranasal rupture of one of these nerve sheaths would occasion cerebrospinal fluid rhinorrhoea.

It is not believed that raised intracranial pressure is necessary for the development of primary spontaneous cerebrospinal fluid rhinorrhoea. The normal pressure of the fluid is positive in many positions of the head. Further, it is subject to recurring fluctuations. Observation of the fluctuations in intraventricular pressure (O'Connell, 1953) showed recurring rhythmic fluctuations of cerebrospinal fluid pressure averaging $80 \mathrm{~mm}$. cerebrospinal fluid every fèw seconds. These observations have more recently been checked using an electromanometer and a similar average total recurring variation in pressure confirmed. It is believed that the deep excavations of the cranial vault which are occupied by arachnoid granulations are produced by the cerebrospinal fluid pulse. When this pulse gains access to the lamina cribrosa it could also produce erosion of tissue here and as a result cerebrospinal fluid rhinorrhoea is established spontaneously.

\section{SUMMARY}

Cases of spontaneous cerebrospinal fluid rhinorrhoez are classified into primary and secondary groups, and the literature is reviewed. Two personal cases are described. A hypothesis to explain the occurrence of primary spontaneous cerebrospinal fluid rhinorrhoea is put forward.

I am indebted to the Department of Medical Illustration St. Bartholomew's Hospital, for the drawings of Mr. P Cull, and for the photographs of the radiographs by. Mr. Tredennick. I must also express my gratitude t $\vec{F}$ Dr. B. Smith and Dr. Gordon Canti for the preparation and examination of the sections of the cribriform area.

\section{REFERENCES}

Adson, A. W. (1941). Proc. Mayo Clin., 16, 385.

Cloward, R. B., and Cunningham, E. B. (1947). J. Neurosurg., 4, 519 Coleman, C. C., and Troland, C. E. (1947). Ann. Surg., 125, 718 . is Johnston, W. H. (1926). Ann. Otol (St. Louis), 35, 1205.

Key, A., and Retzius, J. (1875). Studiens in der Anatomie des Nerver $\vec{P}$ systems und des Bindgewebes (Stockholm).

Le Gros Clark, W. E. (1929). Ministry of Health Reports on Publie Health and Medical Subjects No. 54. H.M. Stationery Offices London.

Locke, C. E. (1926). Arch. Neurol. and Psychiat. (Chic.), 15, 309.

Love, J. G., and Gay, J. R. (1947). Arch. Otolaryng., 46, 40.

Magendie, F. (1842). Recherches physiologiques et cliniques sur î̀ liquide céphalo-rachidien ou cérébro-spinal. MequigngnMarvis, Paris.

Miller, C. (1826). Trans. med.-chir. Soc. Edinb., 2, 243.

O'Connell, J. E. A. (1953). Brain, 76, 279.

Rand, R. W. (1953). Bull. Los Angeles neurol. Soc., 18, 74.

Schwalbe, Schultze's Arch. II. s. 87. Centrlbl. \& Med. W. 1869. Thomson, St Clair (1899). The Cerebro-spinal Fluid; its spontaneouss escape from the nose. Cassell, London.

Weed, L. H. (1914). J. med. Res., 31, 21, 51, 93.

Wessels, A. (1939). Ann. Otol. (St. Louis), 48, 528. 